\title{
Polysèmes
}

Revue d'études intertextuelles et intermédiales

\section{Les voyages de Gulliver : Jonathan Swift et Angela Carter}

\section{Marie-Pascale Buschini}

\section{(2) OpenEdition}

Journals

Édition électronique

URL : http://journals.openedition.org/polysemes/1625

DOI : 10.4000/polysemes. 1625

ISSN : 2496-4212

Éditeur

SAIT

Édition imprimée

Date de publication : 1 janvier 2003

Pagination : 127-148

ISSN : 0999-4203

\section{Référence électronique}

Marie-Pascale Buschini, « Les voyages de Gulliver : Jonathan Swift et Angela Carter », Polysèmes [En ligne], 6 | 2003, mis en ligne le 10 janvier 2017, consulté le 19 avril 2019. URL : http:// journals.openedition.org/polysemes/1625; DOI : 10.4000/polysemes.1625

Ce document a été généré automatiquement le 19 avril 2019

Polysèmes 


\title{
Les voyages de Gulliver : Jonathan Swift et Angela Carter
}

\author{
Marie-Pascale Buschini
}

1 Les références intertextuelles de tous ordres foisonnent dans l'œuvre d'Angela Carter : littéraires tout d'abord avec Shakespeare, Poe, Blake, Byron, Hoffmann, Proust, Baudelaire, Sade, Dostoïevski pour n'en citer que quelques-unes; picturales avec entre autres Goya, les surréalistes, Duchamp, Ernst, les préraphaélites; puis musicales avec Wagner, Mozart, Beethoven, et le music-hall ; ou cinématographiques, essentiellement à travers les films américains de la grande époque d'Hollywood, des années trente aux années cinquante. Parmi cette multitude, les passages de Swift ne sont pas, de loin, les plus nombreux, et ils se limitent aux Voyages de Gulliver. Cependant, Gulliver traverse au fil des années pratiquement toutes les œuvres de fiction de Carter: Shadow Dance (1966) ${ }^{1}$, The Magic Toyshop $(1967)^{2}$, Heroes and Villains $(1969)^{3}$, Love $(1971)^{4}$, The Infernal Desire Machines of Doctor Hoffman $(1972)^{5}$, Fireworks $(1974)^{6}$, Nights at the Circus (1984) ${ }^{7}$. Enfin, Swift est également mentionné dans l'essai que Carter consacra à Sade, The Sadeian Woman: An Exercise in Cultural History (1979) ${ }^{8}$.

2 À travers Les Voyages de Gulliver, Swift a eu une influence toute particulière sur l'imaginaire de Carter et un impact non négligeable sur la construction de son univers romanesque - « Eighteenth-century literature evidently struck a particularly responsive chord » ainsi que le fait remarquer Peter Kemp ${ }^{9}$ - même si cela ne se manifeste la plupart $\mathrm{du}$ temps que sous forme d'allusions. En termes de fréquence, le quatrième voyage, au pays des Houyhnhnms, est le plus employé. C'est d'ailleurs le seul dont un épisode entier d'un roman est inspiré (le chapitre 7 de The Infernal Desire Machines of Doctor Hoffman, «Lost in Nebulous Time»). Viennent ensuite les deux premiers voyages, à Lilliput et Brobdingnag, et enfin le voyage à Laputa. Il est intéressant de constater que les différentes parties du monde vers lesquelles Swift dirige Gulliver sont également des lieux privilégiés chez Carter, elle-même grande voyageuse: Brobdingnag touche l'Amérique, où Carter situe l'action de The Infernal Desire Machines of Doctor Hoffman et The Passion of New Eve (1977); Laputa et ses petites îles satellites sont proches du Japon où 
Carter vécut pendant deux ans, et qui sert de cadre à la plupart des nouvelles de Fireworks ; le pays des Houyhnhnms est proche des côtes d'Afrique et de Madagascar, tout comme le pays des Centaures, qu'il a inspiré dans The Infernal Desire Machines of Doctor Hoffman.

3 Le récit de voyage, si populaire au temps de Swift («I have perused several books of travels with great delight in my younger days [...] many fabulous accounts $\left.»^{10}\right)$, dont les différents épisodes s'apparentent parfois au conte ou à la fable - autres genres auxquels Carter s'est vivement intéressée et qu'elle a largement pratiqués ${ }^{11}$ - est également une constante dans les romans de cette dernière. Ses héros, dont l'un possède un exemplaire des Voyages de Gulliver $^{12}$, sont de grands voyageurs dont les pérégrinations et les multiples aventures illustrent ses thèmes favoris : la perte de l'innocence, ou tout au moins le rite de passage de l'adolescent, et la construction de l'identité. Outre la forme plus lapidaire de la nouvelle, Carter reconnait avoir une prédilection pour ce genre romanesque dont elle définit ainsi le but: «to entertain and instruct, [...] a certain eighteenth-century fictional device-the picaresque, where people have adventures in order to find themselves in places where they can discuss philosophical concepts without distractions $»^{13}$. D'autre part, la conception chez Carter de la fonction du roman (" the novel has some role and responsibility in helping to explain experience and making the world comprehensible, even if it's only to the person who's writing it $\left.{ }^{14}\right)$ a, malgré les restrictions qu'elle y apporte, une parenté certaine avec l'ambition de Gulliver présentant le récit de ses aventures, même si Swift, dans son souci d'en prouver "l'authenticité ", semble rejeter la fonction récréative du récit de voyage au profit de sa fonction didactique :

my principal design was to inform, and not to amuse thee. [...] a traveller's chief aim should be to make men wiser and better, and to improve their minds by the bad as well as good example of what they deliver concerning foreign places. (340)

Enfin, le récit de voyage allié à la fable, tel que le pratiquent Swift et Carter en présentant des utopies/dystopies, ces «comic infernos» dont parle Roz Kaveney ${ }^{15}$, permet bien évidemment de porter un regard neuf sur la société et le monde dans lequel nous vivons. Leurs buts naturellement diffèrent, mais leurs préoccupations se rejoignent bien souvent.

Lorsqu'il se trouve en situation d'infériorité physique, à Brobdingnag, ou dans un univers par trop étranger au sien, au pays des Houyhnhnms, Gulliver réagit par ce que l'on pourrait assimiler à un réflexe de survie, en revendiquant sa qualité de créature rationnelle ${ }^{16}$. Pour Swift, l'homme est par nature foncièrement mauvais, bestial. Ainsi, Gulliver attend-il le pire de ses contemporains européens. Mais, comme de nombreux voyageurs de son époque, il redoute également le danger bien réel et beaucoup plus immédiat que représentent les « hommes sauvages » qui peuplent les îles inconnues où se trouvent rejetés les naufragés. En arrivant à Brobdingnag, il craint immédiatement de se faire dévorer ${ }^{17}:$ «[...] for, as human creatures are observed to be more savage and cruel in proportion to their bulk, what could I expect but to be a morsel in the mouth of the first among these enormous barbarians ${ }^{18}$ who should happen to seize me ?» (125).

Cependant, le premier contact avec les Yahoos au pays des Houyhnhnms est bien plus éprouvant encore pour Gulliver, car il ne met pas en cause son intégrité physique, mais son identité même: "My horror and astonishment are not to be described, when I observed, in this abominable animal, a perfect human figure » (276). Tout l'épisode des Houyhnhnms et des Yahoos retrace les efforts désespérés de Gulliver pour se définir uniquement comme une créature de la Raison, un Houyhnhnm, et nier définitivement l'évidence physiologique de son appartenance à la race des Yahoos. Il ne parvient pas à 
résoudre cette douloureuse tension entre les deux pôles de la nature humaine: il ne cherche pas à dépasser l'apparence en ce qui concerne les Yahoos, mais imite le comportement et jusqu'aux attitudes physiques des Houyhnhnms. Il adopte totalement leur point de vue et leurs convictions ${ }^{19}$ " rationnelles ». Il est tellement persuadé que les Yahoos sont des animaux, des êtres inférieurs, qu'il n'éprouve pas la moindre hésitation, pas le plus petit scrupule à utiliser des peaux de Yahoos pour se confectionner des vêtements, des chaussures, un auvent, une voile, des rames, et jusqu'à leur graisse pour calfater son bateau ${ }^{20}$. Cela le conduit, à son retour en Angleterre, à faire preuve d'une misanthropie extrême, et donc à se montrer tout aussi odieux pour ses contemporains et sa famille que les Yahoos qu'il abhorre. En préférant la compagnie des deux chevaux qu'il entretient dans ses écuries, il perd son identité, renonce en fait à son humanité en poussant sa démarche jusqu'à l'absurde et l'intolérable.

7 Les thèmes de la raison, la civilisation, la culture, la bestialité, l'animalité, la nature sont également très présents chez Carter. Cependant, son approche prend en compte l'influence de Rousseau ${ }^{21}$, de Sade, les découvertes de l'anthropologie et de la psychanalyse. Elle démystifie férocement le mythe rousseauiste de l'innocence et de la bonté de l'homme à l'état de nature : «Myths deal in false universals, to dull the pain of particular circumstances $»^{22}$. Elle voit le monde en termes de rapports de pouvoir, essentiellement entre carnivores et herbivores ou, dans l'optique féministe, entre hommes et femmes. La violence inhérente à ce type de relations est inévitable, seules varient ses manifestations, et il est vital, sinon de faire partie des forts, tout au moins d'apprendre à composer avec eux. La glorification apparente de la raison poussée jusqu'à l'absurde, tout comme chez Swift d'ailleurs, sert par contre chez elle à valoriser l'imaginaire, l'instinctif, l'inconscient et ainsi l'animalité - mais non la bestialité - devient une valeur positive et salvatrice ${ }^{23}$.

8 Lorsque Marianne, l'héroïne de Heroes and Villains déclare : «the Barbarians are Yahoos but the Professors are Laputans $»^{24}$, l'assimilation implicite chez Swift de la raison et de la civilisation est tournée en dérision par le téléscopage des deux voyages de Gulliver : les Professeurs de Carter sont les héritiers spirituels du siècle des lumières, mais dans le monde dévasté qui est le leur, leur attachement passéiste à des valeurs caduques est stérile. De plus, leur obstination à perpétuer un savoir désormais inutile les amène à oblitérer toute la dimension émotive, instinctive, inconsciente de l'homme. La perfection glacée des Houyhnhnms ${ }^{25}$ est alliée chez eux à l'incapacité des Laputans à appliquer la théorie pour affronter les réalités matérielles et quotidiennes du monde.

9 Les Barbares-Yahoos, par contre, ne sont pas abjects mais pathétiques. Ils vivent misérablement dans l'hostilité de la jungle post-nucléaire, dans des conditions matérielles très précaires, sous la coupe d'un ex-professeur de sociologie, disciple de Lévi-Strauss reconverti en chaman, qui s'applique à recréer une mythologie et une religion à leur usage. Contrairement aux Yahoos de Swift, qui conservent toutes les perversions de l'homme civilisé en régressant à l'état de nature, ils vivent dans une société structurée, mais qu'ils ne maîtrisent pas et dont ils sont d'une certaine façon victimes ${ }^{26}$. Ils ont pourtant préservé les valeurs humaines essentielles. Ce ne sont pas des êtres dégénérés, mais des hommes en détresse qui s'appliquent à survivre du mieux qu'ils peuvent. Ainsi le commentaire cruel de Donnally sur Jewel, « he could be the Messiah of the Yahoos ${ }^{27}$, met en évidence sa propre arrogance et la futilité de sa domination sur eux ainsi que leur vulnérabilité, due en grande partie à l'ignorance dans laquelle il les tient (il refuse de leur 
apprendre à lire, par exemple) et par laquelle il asseoit et parvient à maintenir son pouvoir.

10 L'animalité prend aussi chez Swift la dimension purement biologique des fonctions naturelles et de la satisfaction des besoins physiques, qu'il évoque à loisir, et que l'on retrouve chez Carter.

11 La nourriture, le détail des menus et de la préparation des mets, tiennent une grande place. Cependant, chez Swift, ce sont des moyens de souligner la relativité des perceptions et des appétits - Gulliver mange autant que 1728 Lilliputiens, est écœuré par les quantités de nourriture avalées par les géants, et admiratif devant les plats en forme d'instruments de musique ou de figures géométriques de Laputa $(58,80,145,202-203)$ alors que chez Carter, manger est soit un acte d'oppression qui affirme les instincts carnivores ou dominateurs, soit une occasion conviviale qui exprime la sensualité et la chaleur des sentiments ${ }^{28}$.

Il n'y a pas loin du carnivore et du prédateur au cannibale, et le pas est allègrement franchi à la fois par Swift et par Carter. Gulliver fait mine de vouloir dévorer les Lilliputiens qui le tourmentaient et que l'empereur lui a livrés, et craint lui-même, juste retour des choses, d'être dévoré par les géants de Brobdingnag ${ }^{29}$, d'être la proie de leur chat puis d'un rat $(66,125,132)$. Chez Carter, la terreur ancestrale de la dévoration existe vis-à-vis des personnages prédateurs essentiellement.

Mais elle va parfois au-delà du fantasme du parent ogre : Desiderio échappe de justesse au meurtre et au cannibalisme rituel chez les «River People», et le Comte finit effectivement dans la marmite de la tribu du chef noir ${ }^{30}$. Walser, héros de Nights at the Circus, joue quant à lui le rôle du poulet ou de la dinde («the human chicken») dans un atroce numéro de cirque, "the clowns' Christmas dinner ", où un clown dément et homicide le poursuit sur la piste, un énorme couteau à la main, faisant éclater brutalement la frontière ténue qui sépare le spectacle de la vie ${ }^{31}$. Rappelons cependant que Swift dans son Modest Proposal n'avait pas non plus hésité devant cette transgression.

Enfin, restent le stade final et les accidents du cycle de la digestion. Swift est réputé pour la complaisance avec laquelle il évoque les excréments. Gulliver raconte par exemple avec force détails la panique des Lilliputiens lorsqu'il manque de les noyer en urinant, et comment il éteint l'incendie du palais par le même moyen; puis le sans-gêne des dames de Brobdingnag dans la même situation $(60,131-132,158)$. On ne compte pas le nombre de notations à caractère scatologique $(270,301,309-310,313$, etc.), des mesures pratiques prises par les Lilliputiens pour nettoyer sa maison chaque matin après qu'il a fait ses besoins, aux études sur les excréments de l'académie de Lagado, en passant par sa chute dans une bouse de vache monstrueuse à Brobdingnag $(64,164,225)$ et la façon de déjouer les complots à Lagado en examinant le régime alimentaire et les selles des suspects (235). La coprophagie, l'essentiel de la médecine des Yahoos, les lavements ${ }^{32}$ et les vomissements occupent également une place de choix.

Il n'est pas question ici de faire une analyse de l'obsession stercorale de Swift, mais simplement de montrer en quoi elle se rapproche des préoccupations féministes de Carter qui, d'ailleurs, ne dédaigne pas non plus ce type de détails. Les Barbares de Heroes and Villains se soulagent là où ils se trouvent dans la demeure en ruine qui les abrite. Dans The Passion of New Eve (1977) les femmes de Zero souillent la maison de Tristessa en urinant sur le sol. Dans Nights at the Circus (1984), Fewers lâche des pets sonores, Walser urine bruyamment derrière un paravent, et se fait abreuver de sa propre urine par le 
chaman. On découvre dans Wise Children que l'urne en forme de buste de Shakespeare, contenant de la terre de Stratford, et transportée religieusement jusqu'à Hollywood, a été utilisée par le chat pour faire ses besoins pendant le voyage, etc. Pourtant, chez Carter, ces épisodes relèvent de la dérision, ou sont, comme le langage cru qu'elle affectionne, l'expression de la vitalité rabelaisienne de ses personnages.

Dans sa préface à The Sadeian Woman, Carter définit ainsi son approche de l'œuvre du divin marquis :

This book, which takes as its starting point of cultural exploration the wealth of philosophically pornographic material about women that Sade provides, is an exercise of the lateral imagination. Sade remains a monstrous and daunting cultural edifice; yet I would like to think that he put pornography in the service of women, or, perhaps, allowed it to be invaded by an ideology not inimical to women. And give the old monster his due..$^{33}$

Elle aborde naturellement la coprophilie de Sade et de ses libertins, mettant en avant son interprétation psychanalytique comme régression au stade anal. Le libertin manifeste ainsi sa domination sur ses victimes par le droit qu'il se donne et qu'il leur retire de contrôler la production et l'évacuation des excréments, de les consommer, de s'y vautrer, et donc d'adopter impunément un comportement infantile ${ }^{34}$.

Plus intéressante cependant semble l'incompatibilité qu'elle met en évidence entre l'idéal de la femme vertueuse, la victime, Justine, contre laquelle elle s'insurge d'ailleurs violemment, et les fonctions naturelles :

They force her to publicly perform excretory activity she has always conducted furtively, in private, as if it were an activity that in itself degraded her, an activity too human, too common to be publicly acknowledged by such a rare creature as herself. (Swift exclaimed in horror: 'Celia shits!' How can it be possible such a precious being, all angel and no ape, should ever do such a thing? God must be very cruel to shatter our illusions so). ${ }^{35}$

19 Ce qui, chez Sade, est transgression jubilatoire, revient - pour le libertin - à refuser de reconnaître l'image idéalisée que présente la femme d'elle-même, la valeur excessive en terme d'économie des relations sociales qu'elle s'accorde au nom de sa vertu - princesse lointaine, perfection éthérée - pour la ravaler au rang d'objet ordinaire, vulgaire, de simple marchandise soumise aux lois du marché. C'est ce que Carter, reprenant les termes employés par Sade, appelle «la profanation du temple» («the desacration of the temple »).

Swift, par contre, s'il évoque avec complaisance les activités d'excrétion de Gulliver (un homme) et des êtres inférieurs assimilés à des animaux (les Yahoos), ne peut les envisager sans frémir chez des femmes (les dames d'honneur de la cour de Brobdingnag) et sans horreur quand il s'agit de l'être aimé. Il semble donc que la relation de Gulliver/Swift avec les femmes soit sous le signe d'un rapport aux excréments inverse de celui de Sade: admettre que les femmes puissent déféquer revient à renoncer à l'idéal de féminité qu'il a construit $^{36}$, ce qui l'épouvante. Le seul temple qu'il puisse profaner de ses excréments est celui des Lilliputiens où il est logé. Il devient dès lors difficile d'envisager sereinement la sexualité, cette autre fonction naturelle essentielle.

21 Gulliver présente le sexe comme une perversion ou un moyen de promotion sociale, par la prostitution (245). Les relations sexuelles ravalent l'homme (et le Lilliputien) au rang de l'animal ${ }^{37}$. Sa perception des femmes en tant qu'êtres de chair et dans leurs fonctions biologiques est donc terrifiante : la maternité, avec la vision de la nourrice allaitant un enfant à Brobdingnag lui donne littéralement la nausée ${ }^{38}$. Les seins des dames d'honneur 
de la cour, qui se dévêtent devant lui, le déshabillent comme une poupée et l'assoient, par jeu, à cheval sur leurs mamelons sont, contrairement à ce que l'on pourrait attendre, dépourvus de tout attrait érotique. "I was much disgusted", note-t-il, d'autant que l'odeur de leur peau - autre élément traditionnellement érotique - lui est insupportable (157).

Lorsqu'il arrive chez les Yahoos, sa situation empire : il n'est plus simplement objet de divertissement, protégé du désir des géantes par sa petite taille, mais devient lui-même objet de désir, objet sexuel. Il échappe de peu au viol par une femelle Yahoo, est outré par le fait que la grossesse ne mette pas un frein à leurs appétits de relations sexuelles, et il est soumis à leurs regards concupiscents et à leur propension au voyeurisme. La proposition de castrer les Yahoos, avancée par le conseil des Houyhnhnms, ne semble donc pas l'émouvoir outre mesure (314, 310, 311-312, 320).

Il y a bien entendu un élément de misogynie plus classique, de satire sociale, dans l'évocation des mœurs des Yahoos: coquetterie, mesquinerie et vanité des femelles, promiscuité sexuelle. Mais il n'en reste pas moins que Gulliver éprouve une terreur réelle et bien plus profonde de la féminité : ou bien les femmes sont des géantes toutespuissantes, ou bien ce sont des animaux prédateurs caractérisés par une sexualité débridée, auxquels il assimile sa propre femme à son retour en Angleterre. Dans tous les cas, elles sont repoussantes, menaçantes, et il ne s'envisage face à elles que comme objet, et non sujet, de désir. Il se retrouve paradoxalement dans la situation psychologique du type de la femme-victime chez Sade, dont la vertu appelle toutes les infamies car, et c'est la conclusion de Carter en ce qui concerne Justine :

To be the object of desire is to be defined in the passive case.

To exist in the passive case is to die in the passive case-that is, to be killed.

This is the moral of the fairy tale about the perfect woman. ${ }^{39}$

Gulliver, quant à lui, renonce à toute sexualité, ce qui n'est pas une morale bien réjouissante non plus. Cependant, et, pour paraphraser les termes de Carter, «to give Swift his due ", on trouve également dans Les Voyages de Gulliver un certain nombre d'idées qui ne peuvent déplaire à une féministe : à Lilliput comme à Brobdingnag, les enfants des deux sexes reçoivent la même éducation; les Houyhnhnms pratiquent le contrôle des naissances; et Gulliver constate partout où il passe que le mariage n'est pas une bonne institution. Il dénonce, comme le feront Carter et Simone de Beauvoir, entre autres, le fondement économique du mariage, qui institue une situation de dépendance pour la femme. Même si ses arguments sont parfois différents - sans le bon sens de sa femme, l'époux, rongé par les maladies vénériennes contractées dans sa jeunesse, provoque immanquablement une dégénérescence des familles nobles « unless the wife take care to provide a healthy father among her neighbours or domestics » (304) -, il valorise malgré tout ainsi le rôle de la femme et pourrait ironiquement parvenir à la même conclusion que Carter : " a mother is a biological fact, whilst a father is a movable feast $»^{40}$.

Pourtant, si Carter approuve la critique du mariage, elle ne cautionne pas la misogynie de Swift, et elle récupère pour la détourner, à son profit et à celui des femmes, l'image des Struldbruggs. Gulliver explique que l'immortalité dont ils jouissent est une calamité et non une bénédiction, car elle s'accompagne de dégradation physique, et d'une exacerbation des défauts communs à tous les hommes; et surtout à toutes les femmes : " they were the most mortifying sight I ever beheld, and the women more horrible than the men» (258). Ils sont donc charitablement libérés des liens du mariage lorsqu'ils 
atteignent l'âge de 80 ans. La "Struldbrugg » de Carter, en revanche ${ }^{41}$, vieille femme qui travaille dans une cafétéria, est d'une tout autre nature. Elle est gaie, chaleureuse, maternelle, et la fragilité de l'âge la rend presqu'attendrissante. De plus, elle fait partie des survivants : Morris pense l'avoir tuée, et elle reparaît le lendemain matin comme si de rien n'était. Elle a en quelque sorte conquis son immortalité de haute lutte, et annonce les femmes fortes des romans ultérieurs de Carter, celles qui ont appris à courir aux côtés des tigres.

L'incertitude à laquelle est en proie Gulliver quant à son identité sociale - homme et créature rationnelle en Europe, il devient animal chez les Houyhnhnms - et quant à son identité sexuelle - sa terreur de la féminité ne cache-t-elle pas une incapacité à assumer sa masculinité ? - est au centre de la fiction de Carter et du débat féministe contemporain sur le genre (" gender construction » et « gender identity »).

L'image de la femme renvoyée par la société est perçue comme une construction du système patriarcal. Afin de parvenir à définir la féminité, Carter s'applique à démonter cette représentation, qui fait de la femme un objet, une exhibition, un spectacle ${ }^{42}$. Sa fiction abonde en femmes qui se donnent en spectacle ou que l'on contraint à participer à des spectacles: comme actrices aux côtés de marionnettes (Melanie dans The Magic Toyshop); dans des rituels grotesques (le mariage de Marianne dans Heroes and Villains); comme ambassadrice ou tenancière de maison close (Albertina dans The Infernal Desire Machines of Doctor Hoffman) ; actrice de cinéma (Tristessa dans The Passion of New Eve, Daisy Duck dans Wise Children); comme phénomène de foire et/ou artistes de music-hall (Fewers dans Nights at the Circus, Nora et Dora Chance, dans Wise Children); ou encore à la télévision (Tiffany ou Saskia dans Wise Children), pour ne citer que les principales.

La nécessité de présenter une image conforme aux attentes des hommes dominants, souvent assimilés à des marionnettistes ("puppet masters »), contraint ces femmes à porter, au-delà du maquillage, le masque d'une féminité factice, codifiée, et donc à se comporter en automates, ou en marionnettes dont les hommes pensent tirer les ficelles. La découverte de leur nature, de leur identité et de leur féminité - sujet central des romans -, implique un éloignement, un dépassement ou un rejet des normes fixées. Ainsi, les phénomènes, voire les monstres ${ }^{43}$ (hermaphrodites, travestis et transsexuels, nains, géantes, femmes à barbe, homme anguille, femme squelette, femmes polymastes, vampires etc.) sont légion dans leur entourage et parmi elles, avec cependant une prédilection pour ceux qui soulignent la précarité des rapports de domination, et symbolisent l'ambiguïté sexuelle, signe de la difficulté de définir le genre (" gender »).

Le thème du monstre ("the freak ») $)^{44} \mathrm{a}$ d'ailleurs une résonance intime chez Carter, car lorsqu'elle vivait au Japon elle a personnellement et douloureusement fait l'expérience de cette altérité («otherness ») $)^{45}$ de celui qui, étant transporté dans un monde dont les normes diffèrent, devient objet monstrueux soumis au regard des autres :

In the department store there was a rack of dresses labelled: 'For young and Cute Girls Only'. When I looked at them, I felt as gross as Glumdalclitch. I wore men's sandals because they were the only kind that fitted me and, even so, I had to take the largest size [...]. He told me that when he was in bed with me, he felt like a small boat upon a wide, stormy sea. ${ }^{46}$

La dernière création de Carter dans ce domaine, et la plus réussie, est de loin Fewers, la splendide et plantureuse femme ailée, plus vraie que nature («larger than life »), dont le visage présente des traits nettement swiftiens : «a Brobdingnagian symmetry » (Nights at the Circus, 35). C'est une géante, emblème de la nouvelle femme sur le point de prendre 
son envol à l'aube du vingtième siècle, enfin libérée des contraintes économiques, contrôlant sa fécondité, et donc capable de prendre son destin en main.

Il faut cependant noter que Carter ne s'intéresse pas qu'aux femmes: nombre de ses personnages sont androgynes, et certains de ses héros subissent aussi cette déconstruction de l'identité imposée par la société, puis cette reconstruction d'une nouvelle identité qui permet, en se trouvant, de trouver l'autre: Walser doit être dépouillé de son être antérieur comme d'une coquille ("hatched out of the shell of unknowing ", Nights at the Circus, 294) avant de pouvoir retrouver Fewers.

Pour en revenir à Gulliver, outre sa perception des femmes comme objets de contemplation, il se trouve lui aussi dans la situation de celui/celle qui est spectacle, et, curieusement, il l'évoque dans des termes très voisins de ceux qu'emploie Carter. À Lilliput, son gigantisme fascine, mais le laisse en position relativement confortable face à ceux qui l'observent. De même, lorsque Carter compare un de ses personnages à Gulliver à Lilliput, elle invoque une inversion du rapport de force. C'est le cas par exemple pour Melanie (The Magic Toyshop, 86) devant l'arche miniature construite par Uncle Philip où sont représentés tous les membres de la famille : elle comprend qu'il n'est tout-puissant que dans la mesure où ils acceptent de se laisser dominer par lui ${ }^{47}$. Mais les données de départ n'étaient pas celles des Voyages de Gulliver: les héroïnes de Carter partent de Brobdingnag (où elles sont minuscules, dominées) pour arriver à Lilliput (où elles sont géantes, maîtresses d'elles-mêmes), alors que Gulliver passe d'Angleterre (où il est dans la norme, dans un rapport d'égalité) à Lilliput puis à Brobdingnag.

Par contre, à Brobdingag, il est tellement minuscule que même le nain de la cour est un géant pour lui et le tourmente (147). Il est perçu comme un lusus naturae (143), expression employée également par Carter au sujet de Fewers (Nights at the Circus, 161), et le fermier qui l'a découvert l'exploite en l'exhibant dans des foires (136-137), comme les phénomènes de Carter. Enfin, lorsque l'empereur le voit pour la première fois, il ne peut croire qu'il a devant lui un être vivant : « [the Emperor] conceived [he] might be a piece of clock-work (which is in that country arrived to a very great perfection), contrived by some ingenious artist» (142). Ceci rappelle étrangement le slogan de Fewers (« is she fact or is she fiction?»), et évoque les nombreux automates qui traversent les romans de Carter. Pour ne citer que deux exemples, les prostituées exhibées dans des cages dans la maison close de The Infernal Desire Machines of Doctor Hoffman («they were sinister, abominable, inverted mutations, part clockwork, part vegetable and part brute», 132). Une rumeur laisse enfin entendre que Fewers elle-même pourrait être une construction artificielle ( not a woman at all but a cunningly constructed automaton made up of whalebone, india-rubber and springs ", Nights at the Circus, 147).

Pourtant, même si la remise en cause et la (re)construction de l'identité rapprochent Swift de Carter, Gulliver est moins heureux que les héroïnes de cette dernière, car il ne fait pas face à ses monstres mais les fuit, et aboutit à une impasse.

Les variations de point de vue qui font partie de l'univers de Swift et de Carter rapprochent également leurs œuvres. On passe de l'énorme au minuscule pratiquement sans transition, par une série étourdissante de zooms avant et arrière qui amènent le lecteur à prendre brutalement conscience de la relativité des perceptions, et de la précarité des situations habituellement acceptées comme stables. Chez eux, ainsi que le proclame le docteur Hoffman : « only change is invariable » (96). 

univers hors normes: les nains et les géants dominent la terre; les animaux - les Houyhnhnms, mais aussi les chimpanzés de Nights at the Circus qui rediscutent leur contrat avec le directeur du cirque et quittent leur dresseur ivrogne - se comportent plus rationnellement que les hommes; les interdits - cannibalisme, inceste - sont transgressés; les zones d'ombre - scatologie, volonté de pouvoir, fantasmes - sont exposées; les normes sociales et morales sont remises en cause - à Lilliput, les enfants n'ont aucun devoir envers leurs parents et les dignitaires briguent leurs postes par des exercices de cirque, le patriarcat est malmené et les sociétés primitives supplantent la nôtre chez Carter. Pourtant, chacun de ces mondes est un miroir pour le héros, qui lui renvoie une des multiples images possibles de lui-même. Ainsi, Gulliver se retrouve dans la situation des Lilliputiens à Brobdingnag, et les héros de Carter contemplent et évaluent avec leurs doubles androgynes les différents masques qu'ils sont amenés à porter.

La construction du récit de voyage comme une série d'épisodes distincts en fait une juxtaposition de petits contes successifs, où l'extraordinaire devient ordinaire. Ainsi que l'explique Jacqueline Reymond: "Les Voyages de Gulliver oscille constamment entre le récit de voyage et le conte merveilleux et renforce l'hypothèse de Propp que tout récit est mouvement entre deux équilibres semblables mais non identiques $»^{48}$. Chez Carter comme chez Swift, ces contes introduisent en outre une dimension surnaturelle ou fantastique. Carter a largement puisé dans la tradition du conte populaire (The Bloody Chamber, The Courtship of Mr Lyon, The Tiger's Bride, Puss-in-Boots, The Company of Wolves, etc.). Jacqueline Reymond remarque pour sa part que dans Les Voyages de Gulliver, «le merveilleux exotique cède le pas au merveilleux pur. Dans ces pays de songes, l'imaginaire est roi et la tradition des contes de fées à la Perrault, reprise $»^{49}$.

38 Le « Gothic » est également présent chez Carter, à la fois dans les atmosphères (The Magic Toyshop, Heroes and Villains, Love, Fireworks), et dans ses personnages (vampires, fantômes, apparitions, loups-garous). L'expérience cauchemardesque de Gulliver et des géantes, les Struldbruggs immortels, et surtout Glubbdubdrib (" the Island of Sorcerers or Magicians ", (238 et sq), peuplée de spectres sont de la même veine.

39 Enfin, les expériences démentes menées à l'académie de Lagado (extraire des rayons de soleil de concombres, greffer la tête d'un homme sur un autre) et l'île aérienne de Laputa, mue par un complexe système magnétique, quant à elles annoncent clairement la science-fiction et les multiples savants fous de la littérature fantastique ${ }^{50}$. Ces genres sont aussi familiers à Carter, qui a d'ailleurs participé à plusieurs rencontres et colloques d'amateurs de science-fiction. On trouve dans Heroes and Villains un monde futur, post apocalypse nucléaire, et au cœur des États-Unis ravagés par la guerre civile, une ville futuriste, Beulah, miracle de technologie dans The Passion of New Eve. Enfin, ses « puppet masters",

40 Uncle Philip, le Docteur Hoffman, le Ministre, Mother, le Grand Duc ou Christian Rosencreutz sont autant de savants et d'illuminés aux délires mégalomanes.

41 Cependant, ainsi que le montre Roz Kaveney,

the difference between [Carter's "comic infernos", satirical extrapolations of current trends] and Swift, from whom [they] clearly derive, is one of technique-or, to be more precise, it is the technique of the third book of Gulliver's Travels rather than that of the other three; we are not taken through an endless proliferation of details-strange societies are merely sketched in for our benefit, and we become imaginative tourists there rather than inhabitants. ${ }^{51}$

Polysèmes, 6 | 2003 
Le sentiment qu'avait Carter de son étrangeté, de son altérité, de ne pas avoir sa place, perceptible dans tout son œuvre, lui a donc fait choisir Swift comme l'un de ses modèles ( literary parents $»^{52}$ ). Elle pose le même regard désabusé et ironique que lui sur le monde, et le traduit dans sa fiction par des moyens semblables. Elle est d'ailleurs restée jusqu'à sa mort en 1992 en marge du monde littéraire officiel, qui entreprend maintenant de la canoniser, comme des mouvements féministes qui l'ont parfois violemment attaquée pour son approche peu conventionnelle du sexe et de la pornographie. Cependant, elle n'a pas de l'homme et de la société une vision aussi amère, cynique que Swift. Ses provocations sont largement teintées d'humour et de bienveillance, et il n'est pas toujours aisé de faire la part de la dérision et du sérieux dans ce qu'elle avance. Son ironie n'est ni mordante ni désespérée, et elle est parvenue, après la dureté et le pessimisme de ses premiers romans (Shadow Dance, Several Perceptions, Love), à exprimer la sérénité, la sensualité généreuse et la joie de vivre (Nights at the Circus, Wise Children). Les références à Swift sont d'ailleurs de moins en moins nombreuses à la fin de son œuvre. Elle s'est pour ainsi dire finalement détachée de ce "père littéraire », en trouvant son ton propre dans la maturité.

\section{NOTES}

1. London: Heinemann, 1966 ; réédité sous le titre Honeybuzzard, New York: Simon \& Schuster, 1966 ; puis sous son titre original, London: Virago, 1994.

2. London: Heinemann, 1967 ; London: Virago, 1981.

3. London: Heinemann, 1969 ; Harmondsworth: Penguin, 1981.

4. London: Rupert Hart-Davis, 1971 ; London: Picador, 1988.

5. London: Rupert Hart-Davis, 1972 ; réédité sous le titre The War of Dreams, New York: Bard/Avon Books, 1977 ; Harmondsworth: Penguin, 1982.

6. London: Quartet Books, 1974 ; London: Virago, 1988.

7. London: Chatto \& Windus, 1984 ; London: Picador, 1985.

8. London: Virago, 1979.

9. Peter Kemp, «Magical History Tour, an Interview with Angela Carter », The Sunday Times Magazine, 9 June 1991, 6-7.

10. Jonathan Swift, Gulliver's Travels, Harmondsworth: Penguin, 1974, 340. Toutes les références de pages citées dans le texte se rapportent à cette édition.

11. Voir en particulier le recueil de nouvelles The Bloody Chamber. Carter a également traduit les contes de Perrault, et édité les deux volumes des Virago Books of Fairy Tales, 1990 et 1992.

12. Desiderio, dans The Infernal Desire Machines of Doctor Hoffman.

13. John Haffenden, Novelists in Interview, London/New York: Methuen, 1985, 87.

14. Ibid., 79.

15. Roz Kaveney, « New World Dreams ", in Flesh and the Mirror, ed. Lorna Sage, London: Virago, 1994, 181.

16. Voir par exemple "The farmer by this time was convinced I must be a rational creature » (127) ; « For they looked upon it as a prodigy that a brute animal should discover such marks of a rational creature » $(280) ;$ « ...how I was taught to imitate a rational creature » $(281)$; « The report 
spread of a wonderful Yahoo, that could speak like a Houyhnhnm, and seemed in his words and actions to discover some glimmerings of Reason » (282).

17. II est intéressant que cette crainte ne le touche pas à Lilliput, où, pourtant, sa situation semble bien plus délicate d'entrée: il est prisonnier, réduit à l'impuissance. Les géants apparaissent d'emblée à Gulliver/Swift comme les ogres des contes populaires : cannibalisme et gigantisme sont liés. Les implications psychologiques de cette terreur (l'enfant lilliputien face aux adultes géants) semblent claires.

18. On retrouve des barbares chez Carter: dans Heroes and Villains, la jungle qu'est devenue l'Angleterre après la grande catastrophe nucléaire est peuplée de deux groupes qui s'affrontent, «the Professors » (qualifiés de "Laputans ») et « The Barbarians ", assimilés aux Yahoos.

19. Cette attitude se retrouve également chez Desiderio, héros de The Infernal Desire Machines of Doctor Hoffman, qui adopte non seulement le mode de vie des groupes qu'il rencontre (voir par exemple l'épisode des "River People»), mais se fond totalement à eux, envisageant même, comme Gulliver, de s'installer définitivement parmi eux, et de renoncer à ses attaches et à son identité antérieure.

20. $283,324,330$. Notons cependant, indépendamment des échos que cette façon de traiter ses semblables pourraient évoquer dans notre histoire récente, que Swift ne va pas jusqu'à donner le détail des «procédés de fabrication » de tous ces articles.

21. Swift (1667-1745) publie Les Voyages de Gulliver en 1726; Rousseau (1712-1778) publie sa première oeuvre, le Discours sur les sciences et les arts, en 1750 ; le Discours sur l'origine de l'inégalité en 1755.

22. The Sadeian Woman, op. cit., 6.

23. Voir par exemple les nouvelles de The Bloody Chamber (1979) en particulier "The Tiger's Bride ", réécriture féministe et sensuelle de La Belle et la Bête, où la Belle accepte la Bête, est ellemême métamorphosée en Bête après avoir compris la nécessité d'apprendre à courir aux côtés des tigres (« run with the tigers ») pour survivre dans le monde des carnivores.

24. Heroes and Villains, op. cit., 123.

25. Comme l'ont fait remarquer de nombreux critiques, les Yahoos, malgré tous leurs travers, sont infiniment plus vivants que les Houyhnhnms.

26. Les tours de magie et les transes de Donnally, le chaman, entretiennent par exemple en eux une crainte superstitieuse qui assure le pouvoir et l'autorité de ce dernier.

27. Heroes and Villains, 93. Marianne utilise le même terme (" that Yahoo who raped me »), que Donnally reprend plus bas: "Domiciled as you are among the Yahoos, you might as well be Queen of the midden " (61).

28. Voir par exemple le repas de Noël d'Uncle Philip, tyran domestique, dans The Magic Toyshop, 168-169 ; la façon dont Saskia dévore la carcasse du cygne ou prépare un lièvre dans Wise Children ; ou encore, le repas de Desiderio, Albertina et le Ministre dans The Infernal Desire Machines of Doctor Hoffman, 33 et sq.

29. Carter développe le thème du rapport de domination qui fait percevoir la chair comme viande («meat») dans The Sadeian Woman, en évoquant Minski, le géant cannibale des Cent-vingt journées de Sodome: «cannibalism, the most elementary act of exploitation, that of turning the other directly into a comestible; of seeing the other in the most primitive terms of use » (140).

30. The Infernal Desire Machines of Doctor Hoffman, 91-92, 159-165.

31. Nights at the Circus, 174-178. Lorsque le clown est enfin maîtrisé, il est traîné hors de la piste, sous l'œil de chevaux parents des Houyhnhnms : « a section of high-stepping and contemptuous horses, who could spot a Yahoo when they saw one ". C'est le seul endroit où la référence aux Yahoos est explicitement négative, soulignant la bestialité, l'instinct de meurtre et de destruction du clown Buffo, également désigné sous le nom de « the Lord of Misrule ». 
32. Voir par exemple page 226 la précision de la description des expériences menées à l'académie de Lagado pour le traitement de la colique à l'aide d'un soufflet introduit dans l'anus, qui permet soit de vider les intestins soit de les emplir d'air pour les assécher.

33. The Sadeian Woman, 37.

34. Ibid., 86-88, 147.

35. The Sadeian Woman, 74.

36. Cette obsession n'est pas particulière à Swift ou à son époque : le héros des Rachel Papers (1973) de Martin Amis fait preuve du même désarroi en découvrant le slip souillé de Rachel.

37. «men and women are joined together like other animals, by the motives of concupiscence » (97).

38. «I must confess no object ever disgusted me so much as the sight of her monstrous breast [... ]. It stood prominent six foot, and could not be less than sixteen in circumference. The nipple was about half the bigness of my head, and the hue both of that and the dug so varified with spots, pimples and freckles, that nothing could appear more nauseous » (130). Ce sein monstrueux et menaçant semble tout droit sorti du cauchemar psychanalytique de Woody Allen dans Everything You Always Wanted To Know About Sex But Never Dared To Ask.

39. The Sadeian Woman, 76-77.

40. Angela Carter, Wise Children, London: Chatto \& Windus, 1991 ; édition utilisée : London: Vintage, 1992, 216.

41. Personnage secondaire de Shadow Dance, toujours désigné sous ce nom.

42. Voir par exemple Bakhtin, L'Euvre de François Rabelais et la culture populaire au Moyen Âge et sous la Renaissance, Paris : Gallimard, 1970.

43. Voir par exemple à ce sujet: John Sears, Angela Carter's Monstrous Women, English Studies Series Number 3, Sheffield City Polytechnic, 1992.

44. Elle a d'ailleurs écrit une introduction aux Memoirs of a Midget de Walter de la Mare, Oxford: Oxford UP, 1982.

45. Voir Lorna Sage, Writers and their Works: Angela Carter, Plymouth: Northcote House Ltd., 1994, 29 pour les influences, outre Swift et le Japon, qui ont agi sur Carter.

46. « Fireworks », 7.

47. Voir également dans Shadow Dance, 113 et 159.

48. Jacqueline Reymond, "Le merveilleux et l'universel dans Gulliver's Travels », La Licorne, 22, 1992, 209.

49. Ibid., 214.

50. Ibid., 213.

51. Roz Kaveney, « New World Dreams », op. cit., 181.

52. Lorna Sage, Writers and their Works: Angela Carter, op. cit., 29.

\section{INDEX}

oeuvrecitee Fireworks, Gulliver's Travels, Heroes and Villains, Infernal Desire Machines of Doctor Hoffman (The), Love, Magic Toyshop (The), Nights at the Circus, Sadeian Woman (The), Shadow Dance, Wise Children 